\title{
Pragmatics Research and Non-task Dialog Technology
}

\author{
Emer Gilmartin \\ ADAPT Centre, SCSS, Trinity College Dublin \\ Dublin, Ireland \\ gilmare@tcd.ie
}

\begin{abstract}
Interest is growing in dialog systems which engage users in conversations that are not entirely focussed on the immediate performance of clearly defined practical tasks. Interest is also growing in datadriven methods for dialog system design, with increasing focus on sequence-to-sequence deep learning models, inspired by success in the machine translation sphere. In this position paper, we discuss research on casual conversation or social talk and current methods in design of systems, highlighting areas which need reconciliation.
\end{abstract}

\section{CCS CONCEPTS}

- Human-centered computing $\rightarrow$ Human computer interaction (HCI); Natural language interfaces;

\section{KEYWORDS}

Dialog systems, casual talk

\section{ACM Reference Format:}

Emer Gilmartin and Christian Saam. 2020. Pragmatics Research and Nontask Dialog Technology. In 2nd Conference on Conversational User Interfaces (CUI '20), fuly 22-24, 2020, Bilbao, Spain. ACM, New York, NY, USA, 3 pages. https://doi.org/10.1145/3405755.3406142

\section{INTRODUCTION}

Every student of natural language processing is familiar with Jelinek's 1988 remark 'Whenever I fire a linguist our system performance improves', but perhaps not as familiar with his later remark that 'It is our task to figure out how to make use of the insights of linguists' ${ }^{1}$. Insights from pragmatics in particular should give engineers a basis on which to model spoken interaction.

Social or non-task dialog is a goal for many dialog system designers. For the Alexa Prize organisers, a social bot would 'engage the customer in a conversation about popular topics' Microsoft's Xiaoice, a virtual 18 year old social bot, aims to develop emotional intelligence and social skills, with some users seeing the system as 'a dear friend, even a trusted confidante' [17]. These aspirations evoke the well-defined natural phenomenon of social or casual talk. Such 'talk for the sake of talking' builds and maintains social bonds, entertains, and informs participants of each other's personality, values, feelings and affect $[1,7,10,13,19]$. Recent years have seen

${ }^{1}$ Antonio Zampolli Prize acceptance speech, LREC 2004

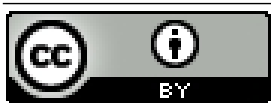

This work is licensed under a Creative Commons Attribution International 4.0 License. CUI '20, July 22-24, 2020, Bilbao, Spain

(C) 2020 Copyright held by the owner/author(s)

ACM ISBN 978-1-4503-7544-3/20/07.

https://doi.org/10.1145/3405755.3406142

\author{
Christian Saam \\ ADAPT Centre, SCSS, Trinity College Dublin \\ Dublin, Ireland \\ saamc@tcd.ie
}

increasing interest in statistical methods for artificial dialog systems. Deep Learning (DL) is used in Natural Language Understanding, Dialog Management (DM) and Natural Language Generation, and increasingly frequently in end-to-end models. Such approaches reduce or eliminate the need for annotation of training data and effectively reduce the complexity of modular dialog systems. However, they are generally much less controllable and require very large data collections to achieve good performance. Recent generic dialog models based on generative pre-training are still hampered by the provenance of the data, from sources such as Reddit threads, which may not be representative of social talk ${ }^{2}$. To mitigate the lack of controllability researchers have proposed various heuristic strategies to achieve more human-like performance, somewhat undermining the hope for true end-to-end systems. Evaluation of artificial chat is made difficult firstly because the notion of good conversation is hard to define and even harder to translate into automatic evaluation criteria.

There are a number of problems inherent with DL approaches (e.g. training/decoding mismatches), but there are also a number of disconnects between the results of research into casual conversation and current design and implementation of non-task systems. Below we describe social talk and outline challenges to modelling such talk.

\section{SOCIAL TALK}

The structure of social casual talk is described as a series of 'subconversations', with greetings, and then interactive smalltalk or chat interleaved with longer stretches of more monologue content-rich talk (chunks) $[8,20]$. It is impossible to model the entirety of such conversations as a series of adjacency pairs [18]. Social bots require both chat and chunks. Chunks are generic (e.g. narrative), and could be modelled separately as of informative contributions by the main speaker, with minimal feedback responses from the interlocutor(s). Chat is not easy to specify, and is often modelled on data drawn from 'information gap' activities where participants try to find out about one another, imposing a structure of successive questionanswer adjacency pairs, or from web data drawn from sources such as Twitter, which, although containing informal language, do not follow the structure of natural casual conversation. Contributions in human chat are a series of statements and sometimes questions, interspersed with backchannels and short comments with little or no additional information [15]. They often include two elements a short coda-like acknowledgement of or comment on the previous speaker's utterance, followed by a new statement or question, as illustrated in the example exchange below. At times of topic exhaustion, conversations have been observed to 'idle', with participants

\footnotetext{
"We note that even with properly filtered Reddit dataset, sometimes our model can still generate moderately toxic/inappropriate responses." https://github.com/microsoft/DialoGPT
} 
producing only short and generic comments (the first element of the two part contributions described above), for a number of turns.

1. A: I saw the match last night.

2. B: Oh. Wasn't Messi great?

3. A: Yeah, but Ronaldo really messed up.

4. B: Yeah. I guess so.

5. A: Yep. He really fluffed that penalty.

6. B: Yeah. But a great match overall.

7. A: Yeah. It was, wasn't it?

8. B: Yeah, good stuff.

9. A: Yeah. Are you golfing much these days?

10. B: Yeah, a bit, but not as much as I'd like.

In the snippet above, the two-part contributions can be seen in turns 2, 3, 5, 6, 9 and 10, with a short 'backward-facing' segment, followed by a 'forward-facing' segment advancing the conversation. Turns 4, 7 and 8 are examples of idling, with low-content segments only. These structures reflect conversation's mixed-initiative nature, where role, power and status differentials between interlocutors are levelled, and participants have equally distributed speaker rights $[5,11]$. They also reflect observations that topics are decided locally and proposable by any participant, rather than following a preset agenda.

\section{CONTROL FEATURES}

In the deep learning dialog modelling community, a number of undesirable and desirable features have been identified, with recent work using these features to control the talk generated [16]. Such features include emphrepetition (undesirable), emphspecificity (desirable), and emphresponse-relatedness (desirable). The origin of these features is unclear, although they are frequently mentioned in the domain.In the light of pragmatics research, their desirability or otherwise is more nuanced. Repetition is often noted as a failing in S2S models, with systems generating short high frequency low information responses [12]. However, as seen above, such responses do occur in talk, but they are only the first half of most turns, (or feedback in chunk phases). Indeed, recent work introducing a two-stage 'sensibility and specificity' human evaluation paradigm [2], intended to avoid 'generic' responses, gives the phrase 'I love tennis' as an example of a non-specific response compared to the preferable specific response 'Me too, I can't get enough of Roger Federer!'. It can be seen that the preferred response actually consists of a backward facing generic 'Me too' and a new contribution 'I can't get enough of Roger Federer!'. The challenge for conversation design is how to generate both elements for realistic social talk. A second consideration related to repetition is that spoken interaction involves alignment between interlocutors, at levels including lexical, syntactic, and prosodic, which is often posited to be necessary for efficient human processing [9]. Once natural conversation gets longer, the level of repetition of words and phrases grows. Thus, a certain level of repetition is necessary and desirable in casual talk. The same factors apply to response-relatedness, as the information rich second element in responses needs to be related to the previous turn while a topic is in progress. However, the introduction of unrelated content for topic changes and even seeming non-sequiters is common in natural talk. Specificity, or the provision of varied language has been identified as a challenge, with measures of word rarity such as Inverse Document Frequency (IDF) used to boost the level of low frequency vocabulary in generated content. However, language in human conversation is generally quite simple, with lower lexical density than in written text $[3,4]$, particularly in face to face environments due to deixis. More diverse language is desirable in longer chunks, or in demonstrations of humour or irony. Again, seeming flaws in current methods actually work well where less specificity is needed, in the first part of contributions, but the more content rich second parts need more specificity.

There are a number of other areas where pragmatic knowledge and deep learning methods do not dovetail. A major challenge is measuring success in casual talk, which is not easily definable at the utterance level or even in a single conversation. Assessment of success, beyond simple measurement of time users spend chatting to the system, is challenging. Systems scoring highly on human evaluation do not always attain high scores on automatic metrics designed to mimic human judgements ${ }^{3}$ [6]. Newer metrics learned by DL may correlate better with human judgments, but are vulnerable to adversarial examples, and thus may be 'gamed' [14]. The channel used in social talk is also important, as human chat is predominantly oral, and very different from formal written text, although recent years have seen the 'conversationalization' of text as more casual or speech-like text is used for informal contact through SMS and social media. Such media have not yet attained true full duplex transmission, and thus turn management differs from face to face communication. There is increasing impetus towards voice interfacing, which will bring further challenges, particularly as social talk has long been considered heavily dependent on prosodic features [1].

\section{CONCLUSIONS}

We have, very briefly, outlined areas where known features of social talk from pragmatics research could pose challenges to deep learning modelling approaches. A fundamental question is whether S2S models as currently implemented are suitable. We have seen that social talk is not a simple series of adjacency pairs, and thus the obvious likeness of adjacency/turn pairs to source and target sentences in MT may not be sufficient justification for S2S and retrieval models. It is also unknown whether the combinatorial explosion of coda and new contribution can be effectively modeled or retrieved from limited data. Concepts emerging from review of generated social conversation, judging the desirability or otherwise of features such as repetition and specificity, may not reflect human social talk. This discrepancy may itself be due to the use of inadequate or artificial data. Although expensive, suitable data are vital to conversational $\mathrm{AI}$, and a worthwhile investment in robust research and development.

\section{ACKNOWLEDGMENTS}

This research was conducted with the financial support of Science Foundation Ireland under Grant Agreement No. 13/RC/2106 at the ADAPT SFI Research Centre at Trinity College Dublin.

\footnotetext{
${ }^{3}$ c.f. ConvAI2, E2E NLG challenges
} 


\section{REFERENCES}

[1] David Abercrombie. 1956. Problems and principles: Studies in the Teaching of English as a Second Language. Longmans, Green.

[2] Daniel Adiwardana, Minh-Thang Luong, David R. So, Jamie Hall, Noah Fiedel, Romal Thoppilan, Zi Yang, Apoorv Kulshreshtha, Gaurav Nemade, Yifeng Lu, and et al. 2020. Towards a Human-like Open-Domain Chatbot. arXiv:2001.09977 [cs, stat] (Jan 2020). http://arxiv.org/abs/2001.09977 arXiv: 2001.09977.

[3] Douglas Biber, Stig Johansson, Geoffrey Leech, Susan Conrad, Edward Finegan, and Randolph Quirk. 1999. Longman grammar of spoken and written English. Vol. 2. Longman London.

[4] Wallace Chafe and Jane Danielewicz. 1987. Properties of spoken and written language. Academic Press.

[5] Christine Cheepen. 1988. The predictability of informal conversation. Pinter London.

[6] Amanda Cercas Curry, Helen Hastie, and Verena Rieser. 2017. A Review of Evaluation Techniques for Social Dialogue Systems. In Proceedings of the 1st ACM SIGCHI International Workshop on Investigating Social Interactions with Artificial Agents (ISIAA 2017). ACM, 25-26. https://doi.org/10.1145/3139491.3139504 eventplace: Glasgow, UK.

[7] R. Dunbar. 1998. Grooming, gossip, and the evolution of language. Harvard Univ Press.

[8] Suzanne Eggins and Diana Slade. 1997. Analysing casual conversation. Cassell.

[9] S. Garrod and M. J Pickering. 2004. Why is conversation so easy? Trends in Cognitive Sciences 8, 1 (2004), 8-11.

[10] Samuel Ichiyé Hayakawa. 1990. Language in thought and action. Houghton Mifflin Harcourt.
[11] John Laver. 1975. Communicative functions of phatic communion. Organization of behavior in face-to-face interaction (1975), 215-238.

[12] Jiwei Li, Michel Galley, Chris Brockett, Jianfeng Gao, and Bill Dolan. 2016. A Diversity-Promoting Objective Function for Neural Conversation Models. 110119. https://doi.org/10.18653/v1/N16-1014

[13] B. Malinowski. 1923. The problem of meaning in primitive languages. Supplementary in the Meaning of Meaning (1923), 1-84.

[14] Ananya B. Sai, Mithun Das Gupta, Mitesh M. Khapra, and Mukundhan Srinivasan. 2019. Re-evaluating ADEM: A Deeper Look at Scoring Dialogue Responses. arXiv:1902.08832 [cs] (Feb 2019). http://arxiv.org/abs/1902.08832 arXiv: 1902.08832.

[15] Klaus P. Schneider. 1988. Small talk: Analysing phatic discourse. Vol. 1. Hitzeroth Marburg.

[16] Abigail See, Stephen Roller, Douwe Kiela, and Jason Weston. 2019. What makes a good conversation? How controllable attributes affect human judgments. arXiv:1902.08654 [cs] (Feb. 2019). http://arxiv.org/abs/1902.08654 arXiv: 1902.08654.

[17] Geoff Spencer. [n.d.]. Much more than a chatbot: China's Xiaoice mixes AI with emotions and wins over millions of fans. https: //news.microsoft.com/apac/features/much-more-than-a-chatbot-chinasxiaoice-mixes-ai-with-emotions-and-wins-over-millions-of-fans/

[18] Scott Thornbury and Diana Slade. 2006. Conversation: From description to pedagogy. Cambridge University Press.

[19] W. Turnbull. 2003. Language in action: Psychological models of conversation. Routledge.

[20] Eija Ventola. 1979. The structure of casual conversation in English. Fournal of Pragmatics 3, 3 (1979), 267-298. 